\title{
Análisis de los contenidos distribuidos por proveedores de publicaciones electrónicas Artículo
}

\author{
Por Blanca Rodríguez Bravo y $\mathbf{M}^{\mathbf{a}}$ Luisa Alvite Díez
}

Resumen: Análisis de los contenidos científicos electrónicos distribuidos por Emerald, Kluwer Online, ScienceDirect, SpringerLink, Taylor \& Francis y Wiley InterScience. Para la evaluación se han establecido tres indicadores: cobertura horizontal, cobertura temática y cobertura vertical. La exploración se ha realizado a partir de las suscripciones mantenidas por la Universidad de León, con la excepción de Kluwer y Taylor \& Francis, para cuyo análisis se ha empleado la consulta web gratuita. Se constata que los proveedores de contenidos electrónicos estudiados son multidisciplinares, si bien ofertan fundamentalmente contenidos de ciencias de la naturaleza, de la salud y de ciencias sociales.

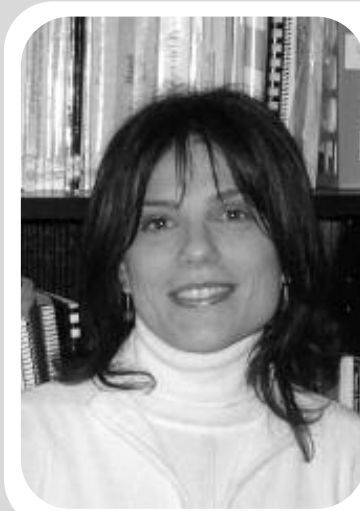

Blanca Rodríguez Bravo es licenciada en geografía e historia por la Universidad Autónoma de Madrid, doctora en historia por la Universidad de León, postgrado en documentación por la Universidad Complutense y en documentación digital por la Universidad Pompeu Fabra. Profesora titular de universidad del Área de Biblioteconomía y Documentación de la Universidad de León. Sus líneas actuales de investigación se hallan relacionadas con la representación de información, la organización del conocimiento y el estudio de interfaces de usuario. Ha publicado dos libros y varios trabajos relacionados con estas líneas en diferentes congresos y revistas.

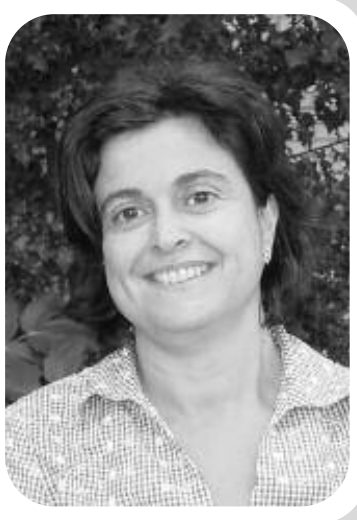

Palabras clave: Cobertura informativa, Evaluación, Proveedores de contenidos electrónicos, Publicaciones electrónicas, Emerald, Kluwer Online, ScienceDirect, SpringerLink, Taylor \& Francis, Wiley InterScience.

Title: Analysis of scientific contents distributed by electronic publications providers

Abstract: Analysis of the scientific content distributed electronically by Emerald, Kluwer, ScienceDirect, SpringerLink, Taylor \& Francis and Wiley InterScience. Three indicators were established for the evaluation: horizontal, thematic, and vertical coverage. The study was performed based on the subscriptions held by the University of Leon (Spain), with the exception of Kluwer and Taylor \& Francis for which the analysis was done via free web access. These electronic suppliers are multidisciplinary, though the content offered is fundamentally limited to the areas of natural sciences, health sciences, and social sciences.

Keywords: Information coverage, Evaluation, Electronic content providers, Electronic publications, Emerald, Kluwer Online, ScienceDirect, SpringerLink, Taylor \& Francis, Wiley InterScience.

Rodríguez, Bravo, Blanca; Alvite Díez, $M^{a}$ Luisa. "Análisis de los contenidos distribuidos por proveedores de publicaciones electrónicas”. En: El profesional de la información, 2004, noviembre-diciembre, v. 13, n. 6, pp. 441-449.

\section{Objetivos y metodología}

En los últimos años han comenzado a surgir los primeros estudios sobre distribuidores de revistas electrónicas que se han dirigido mayoritariamente a la valoración del uso de las publicaciones electrónicas por parte de la comunidad académica. Sirva de ejemplo el reciente trabajo de Tenopir (2003) y, en nuestro país, el dirigido por Urbano (2004). Algunos de estos estudios, como los de Pullinger (1999) y Eason, Ri- chardson y Yu (2000), proporcionan información detallada acerca de la influencia de los contenidos (cobertura y relevancia) y de los aspectos técnicos que condicionan el acceso en el uso de los mismos.

Los fines concretos del presente trabajo se sitúan en la evaluación comparativa de la cobertura de la información distribuida por seis paquetes de contenidos electrónicos. Cinco de ellos contratados mayoritariamente por las bibliotecas universitarias españolas 
Las autoras han realizado dos estudios previos (2004) en los que se ha procedido al análisis individualizado de sendos proveedores de contenidos electrónicos, a saber, el paquete ScienceDirect de Elsevier y Wiley InterScience de John Wiley \& Sons

(Emerald, Kluwer Online, ScienceDirect, SpringerLink y Wiley InterScience) y Taylor \& Francis con una oferta de contratación para 2005 en España que se ha considerado relevante para este análisis conjunto.

El estudio se enmarca dentro de un proyecto de investigación financiado por la Universidad de León para los años 2004/2005, cuyo objetivo central se dirige a lograr un conocimiento global de la provisión de información electrónica, su distribución y uso por parte de la comunidad científica que permita a las universidades y a sus bibliotecas la negociación con las grandes multinacionales del sector editorial, fundamentada en criterios objetivos de calidad y uso.

Los análisis mencionados anteriormente nos han permitido contrastar la validez de la metodología propuesta, en la cual se establecen indicadores para evaluar además de la extensión de los contenidos, la arquitectura de acceso, las características de la interfaz y las funcionalidades y servicios de valor añadido. En esta ocasión nos fijamos en los contenidos suministrados, indicador prioritario para la comunidad científica, que ha de conocer de modo fidedigno qué se oferta, a qué temática corresponde, qué cantidad de información se aporta y cuál es su alcance retrospectivo.

\section{«Es preciso reseñar que la co- bertura lingüística de los conte- nidos de todos los proveedores se circunscribe al inglés, con una escasa presencia del ale- mán y el francés»}

Con esta finalidad hemos articulado el proceso evaluativo de extensión de contenidos en tres indicadores:

- Cobertura horizontal: volumen de publicaciones electrónicas y bases de datos.

- Cobertura temática: disciplinas científicas recogidas.

- Cobertura vertical: alcance retrospectivo de los contenidos.

La exploración de los paquetes electrónicos se ha llevado a cabo desde enero a julio de 2004. Para la evaluación nos hemos servido de las suscripciones existentes en la Universidad de León, con la excepción

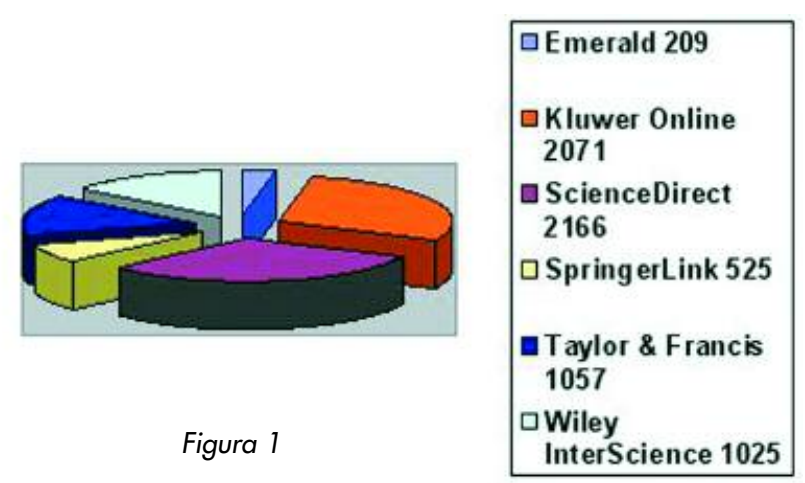

de Kluwer y Taylor \& Francis para cuyo análisis se ha empleado la consulta gratuita.

En el caso del paquete de Elsevier y de Wiley \& Sons se han utilizado los accesos navegacionales alfabéticos, temáticos, por tipos de producto y los listados presentados en la información dirigida al bibliotecario. Por lo que se refiere a Taylor \& Francis se ha empleado el listado provisional de títulos incluidos en el Consorcio Abierto de Taylor \& Francis y Ebsco 2005, remitido a las bibliotecas universitarias en el mes de junio de 2004.

\section{Cobertura horizontal (volumen)}

Se han contabilizado globalmente los contenidos digitales de los distintos suministradores incluyendo publicaciones seriadas, libros electrónicos, obras de referencia, manuales de laboratorio y bases de datos.

Science y Springer presentan un apartado de series de libros, que en este trabajo se han contabilizado como tales. En el caso de Taylor \& Francis, nos hemos ceñido a la oferta enviada por Ebsco que se circunscribe a las publicaciones periódicas y a 10 bases de datos (figura 1).

Kluwer Online y ScienceDirect destacan respecto de otros proveedores por su amplia oferta de contenidos electrónicos. Wiley InterScience resulta ejemplar en la ilustración de las tres plataformas mayoritarias de acceso a los contenidos electrónicos científico-técnicos: revistas digitales, bases de datos referenciales y libros electrónicos. Emerald centra su oferta en las publicaciones seriadas.

Hay que dejar constancia de la sobresaliente oferta de e-books de Taylor \& Francis, que desde su sede web asciende a 8.149 ítems y que, en este momento, no forman parte de los contenidos ofertados para las bibliotecas españolas. Así mismo es notable la oferta de Springer que en sus 30 colecciones de libros contiene 2.537 publicaciones unitarias. Relativamente alta es la densidad de libros electrónicos de Kluwer, (1.210) y, en menor medida, la de Science cuyas 8 series de libros se desglosan en 961 volúmenes; Wiley InterScience dispone de más de 500. Esta realidad obe- 


\section{$\Delta$ \\ docuteca \\ Servicios de Gestión Documental}

C/ Terré, 11-19

08017 BARCELONA

Tel.: 932532201 - Fax: 932532234

Ctra. Antigua Ajalvir, Km. 2,200

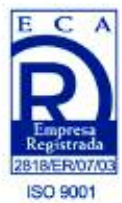

\section{Consultoría sobre la gestión de la información y el conocimiento}

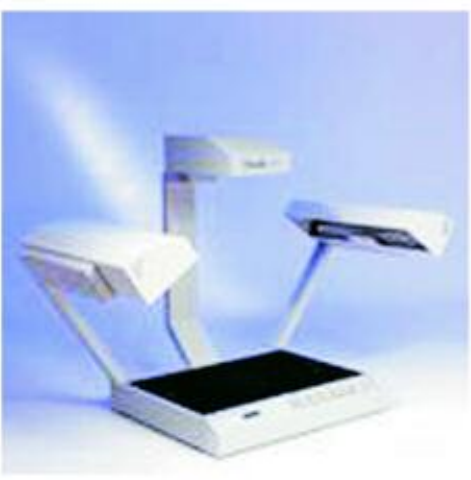

Custodia externa de archivos y soportes informáticos

Digitalización de documentos

Grabación de datos

Tareas auxiliares de archivo

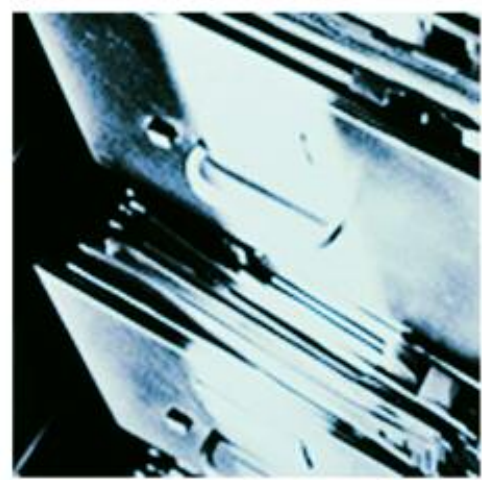

Soluciones para la gestión electrónica de documentos

dece a la política de difusión de las propias editoriales que, disponiendo de los preprints y de la tecnología precisa, aprovechan las ventajas del formato electrónico en la distribución de contenidos. No obstante, las características actuales de las monografías digitalizadas hacen pensar más en un complemento a la labor editorial de la propia empresa que en una apuesta por los e-books, explotando las particularidades que estos productos han de implementar. Nos hallamos ante un sector en pleno despegue que requiere una progresiva aceptación por parte de la comunidad científica.

Destacamos el apartado dedicado a obras de referencia presente en Kluwer, Science y Wiley; este último distribuidor completa sus contenidos con manuales de laboratorio y bases de datos referenciales. ScienceDirect y Taylor \& Francis distribuyen, asimismo, bases de datos.

Emerald, Science y Springer, a partir de los accesos navegacionales, permiten distinguir con facilidad, dentro de los contenidos ofertados en la licencia firmada, aquellos suscritos de los no suscritos por la institución. Los contenidos suscritos facilitan habitualmente el acceso al texto completo, mientras en el resto suelen aportar información meramente referencial o resúmenes. Al carecer la Universidad de León de la licencia de Kluwer Online y de Taylor \& Francis, desconocemos este particular. Por el contrario, resulta difícil delimitar los contenidos a texto completo ofertados en Wiley InterScience acudiendo a los accesos navegacionales que se proponen al usuario.

En la Universidad de León, por lo que se refiere a los e-books y reference works, la oferta se restringe únicamente a las tablas de los sumarios, las introducciones y resúmenes de los capítulos. En el caso de las publicaciones seriadas, destacamos positivamente la profundidad en la digitalización que no se limita a los artículos científicos, ofreciendo, además, editoriales, reseñas, etc.

Es preciso subrayar que la cobertura lingüística de los contenidos de todos los proveedores se circunscribe al inglés, con una escasa presencia del alemán y el francés. Solamente Taylor \& Francis ha incorporado recientemente una publicación española: El profesional de la información.

\section{Cobertura temática}

La identificación de los títulos y su atribución a una única clase temática para valorar la presencia de 
las distintas disciplinas científicas en cada uno de los paquetes de contenidos reviste una gran complejidad.

Salvo en Wiley Interscience, resulta imposible delimitar la correspondencia entre contenidos y categorías temáticas. Sólo este distribuidor facilita por completo la atribución de los ítems a una única categoría. Kluwer mantiene la equivalencia únicamente en el caso de los libros. El resto de los proveedores no permiten conocer la clase principal de adscripción de cada contenido, y es muy frecuente la ubicación del mismo título en diversas clases.

Entendemos que el anclaje de las publicaciones en diversas categorías está diseñado para facilitar al usuario la exploración, pero sería deseable que se facilitase al bibliotecario una organización temática no redundante, a la espera de que se arbitre un sistema que favorezca el acceso sin dispersar contenidos o crear expectativas confusas.

Por la razón expuesta, el recuento por categorías de Emerald, Kluwer Online,

\begin{tabular}{|l|c|}
\hline Kluwer Online categories & Website \\
\hline Arts & 3 \\
\hline Astronomy/Astrophysics/Space science & 22 \\
\hline Biological sciences & 207 \\
Business administration & 70 \\
Chemistry & 140 \\
\hline Computer and information science & 175 \\
\hline Earth sciences & 80 \\
\hline Economics & 126 \\
Education & 89 \\
Electrical and electronic engineering & 68 \\
\hline Engineering & 109 \\
\hline Environmental sciences & 80 \\
\hline Humanities & 41 \\
\hline Law & 38 \\
\hline Linguistics & 35 \\
\hline Materials science & 69 \\
\hline Mathematics & 157 \\
\hline Medicine & 254 \\
\hline Operations research/Management science & 42 \\
\hline Philosophy & 134 \\
\hline Physics & 78 \\
\hline Psychology & 212 \\
\hline Social sciences & 155 \\
\hline
\end{tabular}

Tabla 2

\begin{tabular}{||l|c||}
\hline \multicolumn{1}{|c|}{ Emerald categories } & Website \\
Accounting, auditing \& accountability journal & 9 \\
\hline Continuing professional development & 21 \\
\hline Education & 6 \\
\hline Engineering \& materials science & 17 \\
Environmental management & 4 \\
Food \& nutrition & 3 \\
\hline General management & 21 \\
\hline Healthcare management & 10 \\
\hline Hospitality \& services management & 6 \\
\hline HR management & 25 \\
Information management & 14 \\
\hline Library collection development \& management & 10 \\
\hline Library management \& information services & 16 \\
\hline Library technology & 18 \\
Logistics \& supply chain management & 7 \\
\hline Marketing & 15 \\
\hline Operations \& production management & 13 \\
\hline Police management & 3 \\
\hline Public sector management & 13 \\
\hline Quality management & 14 \\
\hline The built environment & 7 \\
\hline Training & 8 \\
\hline
\end{tabular}

Tabla 1

ScienceDirect, SpringerLink y Taylor \& Francis supera ampliamente el total real que figura en el indicador de cobertura horizontal.

A continuación se muestran los datos que cada uno de los proveedores presenta en su sede web. Resulta tal vez desmesurado el empleo de 22 categorías para un conjunto no excesivo de ítems como el de Emerald (tabla 1). Parecen, en general, muy específicas las etiquetas utilizadas, lo que dificulta la delimitación de sus contenidos. Obsérvese que 12 categorías contienen el término "management". La gestión es el sema que mejor define los contenidos de este distribuidor orientado además hacia la economía, la educación y la biblioteconomía.

En el caso de Kluwer (tabla 2) las categorías temáticas no son equivalentes para publicaciones seriadas y libros. Dada la imposibilidad de establecer equivalencias perfectas, procedemos a mostrar en la tabla únicamente las categorías que la editorial emplea para las revistas y en las que es manifiesto un alto nivel de redundancia. Como en el distribuidor anterior, consideramos elevado el número de clases temáticas que, en este caso asciende a 23 , así como escasamente equilibradas las etiquetas que las representan; en ocasiones demasiado genéricas, como Social sciences, y, en otras, de gran especificidad como Arts, Electrical 


\begin{tabular}{|l|c|}
\hline ScienceDirect categories & Website \\
\hline Agricultural and biological sciences & 177 \\
\hline Arts and humanities & 39 \\
\hline Biochemistry, genetics and molecular biology & 297 \\
\hline Business, management and accounting & 126 \\
Chemical engineering & 84 \\
\hline Chemistry & 137 \\
\hline Computer science & 141 \\
\hline Decision sciences & 46 \\
Earth and planetary sciences & 118 \\
\hline Economics, econometrics and finance & 107 \\
\hline Energy & 56 \\
\hline Engineering & 216 \\
\hline Environmental science & 100 \\
\hline Immunology and microbiology & 103 \\
\hline Materials science & 156 \\
\hline Mathematics & 86 \\
\hline Medicine \& dentistry & 536 \\
\hline Neuroscience & 127 \\
\hline Nursing \& health professions & 59 \\
\hline Pharmacology, toxicology \& pharmaceutical science & 92 \\
\hline Physics \& astronomy & 134 \\
\hline Psychology & 155 \\
\hline Social sciences & 190 \\
\hline Veterinary science \& veterinary medicine & 22 \\
\hline
\end{tabular}

and electronic engineering y Operations research/management science. El mayor número de publicaciones seriadas se concentra en las áreas de Medicine, Psychology y Biological sciences. Las categorías intermedias se corresponden con Computer and information science, Mathematics y Chemistry.

Atendiendo a las categorías navegacionales presentadas en la web de ScienceDirect, se evidencia el alto grado de redundancia que resulta en un $60 \%$ superior a la cifra real ya dada en el apartado de cobertura horizontal (tabla 3). Se encuentran posicionadas en primer y segundo lugar respectivamente las categorías de Medicine \& dentistry y Biochemistry, genetics and molecular biology y resulta reseñable, asimismo, la clase temática de Agricultural and biological sciences. A la vista de los resultados es indudable que integra contenidos de ciencias de la naturaleza y la salud fundamentalmente.

Es significativa la distribución de contenidos de ciencias puras (Chemistry; Mathematics; Physics \& astronomy; etc.), ciencias técnicas (Chemical engineering; Computer science; Energy; Engineering; Materials science; etc.) y ciencias sociales (Business, management and accounting; Economics, econometrics and finance; Psychology; Social sciences; etc.).
Parece cuestionable, como en los proveedores anteriores, el alto número de categorías (24), algunas semánticamente muy próximas, que pueden contribuir a confundir al usuario al dispersar en exceso contenidos similares o idénticos. El mismo título se repite en muchos casos en tres y hasta en cuatro categorías.

Asimismo, manifiestan inconsistencia las etiquetas que las representan, muy extensas en algún caso (Social sciences) y muy específicas en la mayoría de ocasiones (Energy; Decision sciences). La especificidad de denominaciones obliga a la utilización de dobles y hasta triples nombres para las etiquetas, lo que no contribuye precisamente a la coherencia del sistema propuesto.

Consideramos adecuado el número de categorías de Springer, 11 en total, que se adapta correctamente a sus contenidos, al igual que las extensas y claras denominaciones de las etiquetas usadas (tabla 4). Únicamente resulta pobre la oferta de la categoría Law. Destacan, por el contrario, los contenidos de Medicine y Life sciences.

Resulta reseñable, paradójicamente, que en este producto sean numerosas las publicaciones seriadas que no se encuentran asignadas a ninguna categoría temática, no estando, por tanto, disponibles en los accesos navegacionales por materias.

En Taylor \& Francis se establecen 21 categorías temáticas que, a su vez se subdividen en subcategorías, salvo Economics and econometrics (tabla 5). Al igual que en ScienceDirect, es notable el grado de redundan-

\begin{tabular}{|l|c||}
\hline Springerlink categories & Website \\
Chemical science & 56 \\
\hline Computer science & 50 \\
\hline Economics & 34 \\
\hline Engineering & 64 \\
Environmental sciences & 46 \\
Geoscience & 63 \\
\hline Law & 6 \\
\hline Life sciences & 143 \\
\hline Mathematics & 81 \\
Medicine & 198 \\
\hline Physics and astronomy & 78 \\
\hline
\end{tabular}

Tabla 4 
Ciencias de la naturaleza

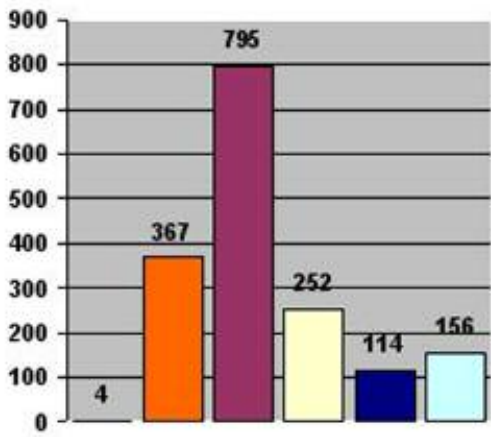

Ciencias sociales

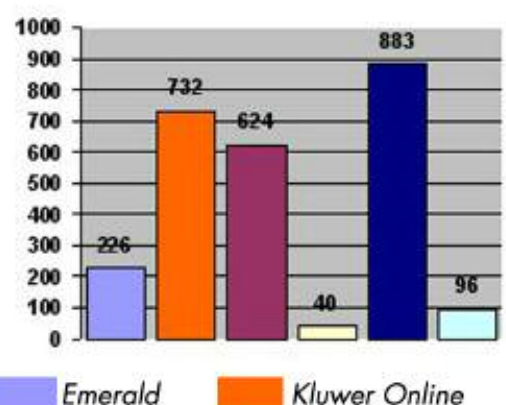

Ciencias puras

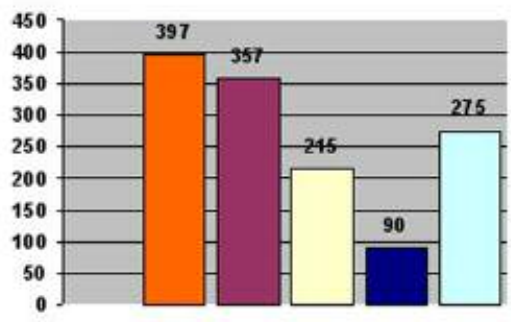

Ciencias de la salud

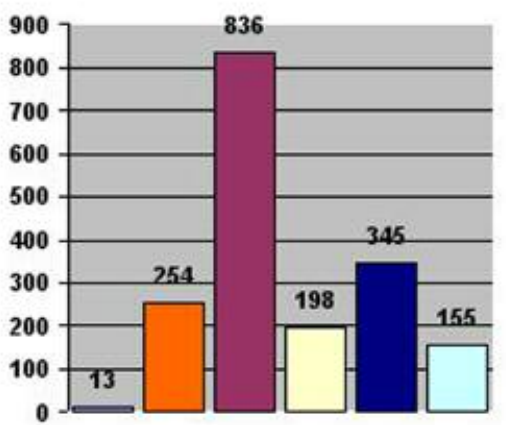

Ciencias técnicas

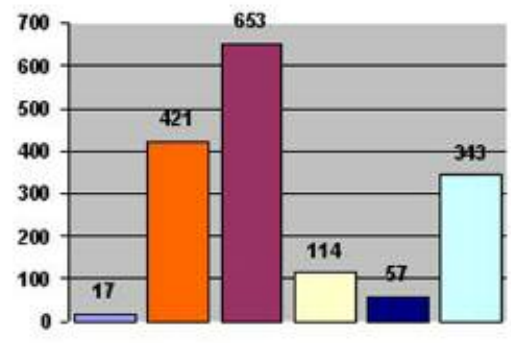

ScienceDirect
Springerlink
Humanidades

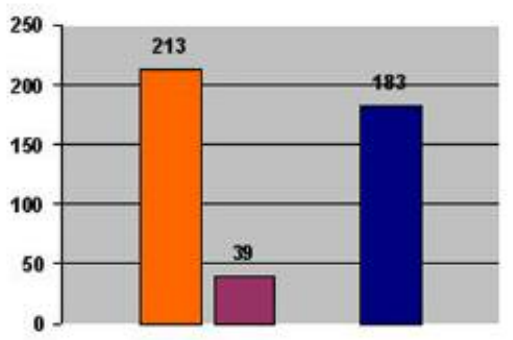

Taylor \& Francis

Wiley InterScience

Figura 2

\begin{tabular}{|l|c|}
\hline Taylor \& Francis categories & Website \\
Agricultural and biological science & 27 \\
\hline Arts \& humanities & 183 \\
\hline Business \& management & 42 \\
\hline Chemistry & 22 \\
Computer science & 6 \\
Earth and planetary science & 15 \\
\hline Economics and econometrics & 32 \\
\hline Education & 190 \\
\hline Energy and power & 3 \\
\hline Engineering and technology & 37 \\
\hline Environmental science & 20 \\
\hline Geography & 78 \\
\hline Health science & 330 \\
\hline Life science & 52 \\
\hline Materials science & 11 \\
\hline Mathematics & 23 \\
\hline Neuroscience & 15 \\
\hline Physics and astronomy & 25 \\
\hline Psychology & 136 \\
\hline Science & 20 \\
\hline Social sciences & 405 \\
\hline
\end{tabular}

Tabla 5 cia de la información mostrada en la web de este distribuidor, cercano al $60 \%$, muy acusado entre las categorías Arts \& humanities y Education. Se ha observado que las clases no son coincidentes con las asignadas para los libros electrónicos.

Destacan, por el número de contenidos, las categorías de Social sciences y Health science. Ambos ámbitos temáticos son, sin duda, los dominantes en este distribuidor. Los contenidos de ciencias sociales se fortalecen si añadimos aquéllos de las categorías de Education, Geography, Psychology, etc.

Algunas clases podrían agruparse y acomodarse jerárquicamente en subcategorías. Sirva de ejemplo de especificidad la etiqueta de Energy and power a la que se adjudican únicamente tres publicaciones. Subrayamos, igualmente, la escasa consistencia en la distribución de las subcategorías de las clases principales, que incluso en algunos casos se repiten, Anthropology \& archeology y Cотmunication son subapartados tanto de Arts \& humanities como de Social sciences.

Wiley establece 14 categorías temáticas que agrupan contenidos correspondientes a las ciencias sociales, ciencias puras, técnicas y de la naturaleza y de la salud, no recogiendo contenidos de humanidades. Dichas categorías se subdividen jerár- 


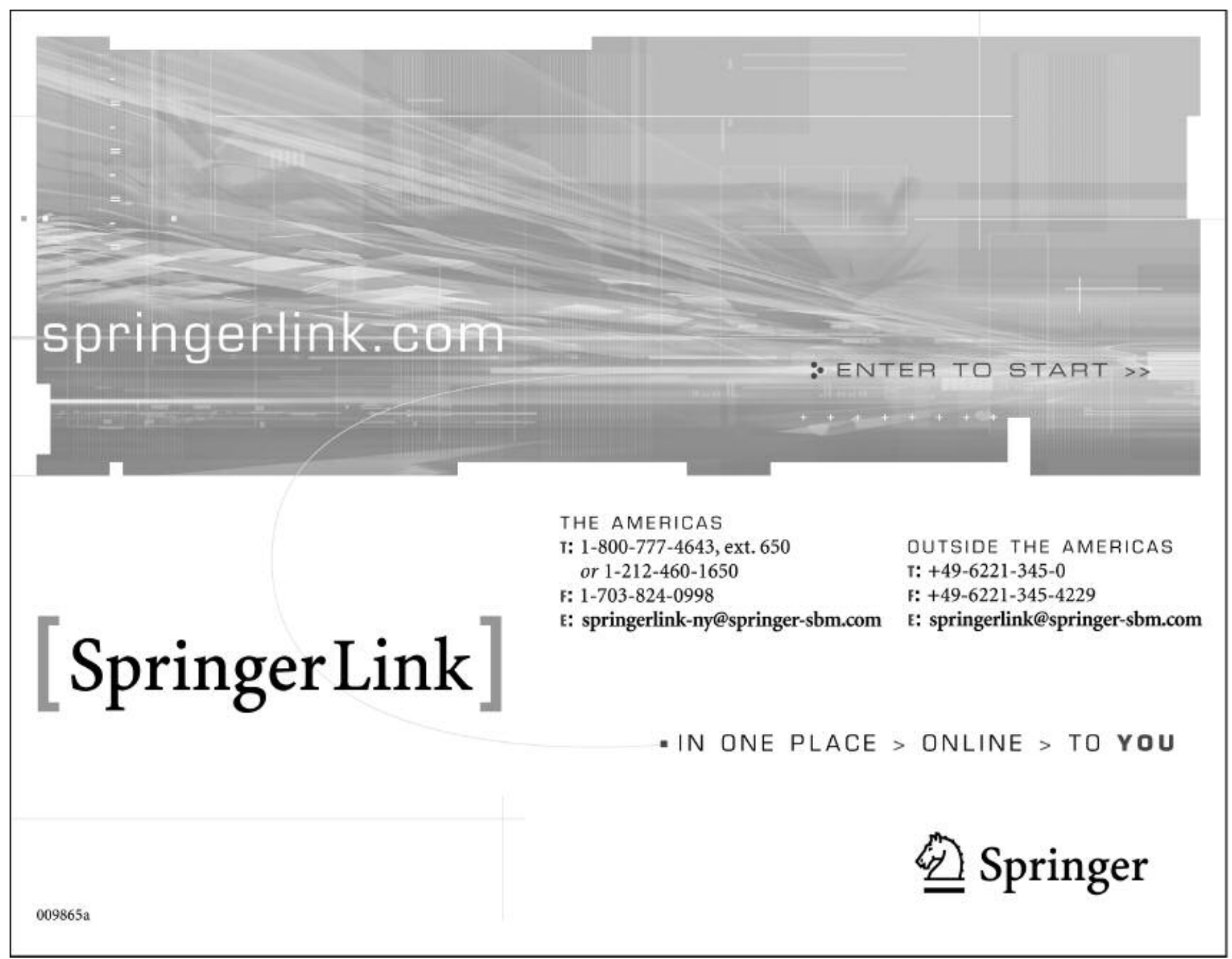

\begin{tabular}{|l|c|}
\hline Wiley Interscience categories & Website \\
\hline Business & 50 \\
Chemistry & 213 \\
\hline Computer science & 17 \\
\hline Earth and environmental science & 28 \\
\hline Education & 11 \\
\hline Engineering & 240 \\
Law & 3 \\
\hline Life sciences & 128 \\
\hline Mathematics and statistics & 49 \\
\hline Medicine & 155 \\
Physics and astronomy & 13 \\
\hline Polymers and materials science & 86 \\
\hline Psychology & 31 \\
\hline Social sciences & 1 \\
\hline
\end{tabular}

Tabla 6 quicamente en subcategorías, visibles desde los accesos navegacionales (tabla 6).

Cuatro clases temáticas: Chemistry, Engineering, Life sciences y Medicine representan casi el $72 \%$ de los contenidos totales. Podemos hablar de tres categorías con un volumen de contenidos intermedio: Business, Mathematics and statistics y Polymers and material sciences. Dada la especificidad de esta última, consideramos de mayor trascendencia los ítems que contiene, en particular el número de libros electrónicos.

Son ciertamente pobres los contenidos de otras cinco categorías: Computer science, Education, Law, Physics and astronomy y Social sciences que representan en su conjunto un $4 \%$ del total.

Parecería más adecuada la agrupación de clases principales y el uso de etiquetas más compensadas, dado que algunas resultan muy genéricas y otras muy específicas. Cabe resaltar la inconsistencia de la categoría de Social sciences con un único ítem bajo un membrete muy extenso semánticamente. En esta clase podrían haberse agrupado los 3 ítems de Law, los 11 de Education e incluso los 50 de Business. 
Con la finalidad de averiguar la adscripción temática de los contenidos distribuidos por las seis empresas analizadas hemos considerado conveniente la agrupación de las categorías en seis áreas temáticas: Ciencias de la naturaleza, Ciencias puras, Ciencias de la salud, Ciencias sociales, Ciencias técnicas y Humanidades. Las categorías Life sciences de SpringerLink, Taylor \& Francis y Wiley InterScience con contenidos mixtos, adjudicables a ciencias de la naturaleza y de la salud se han sumado al primer apartado (figura 2).

Resulta notorio que los proveedores de contenidos electrónicos estudiados son mayoritariamente multidisciplinares; no obstante, han recogido fundamentalmente publicaciones de las áreas de ciencias de la naturaleza, de la salud y sociales. Es escasa, por el contrario, la presencia de las humanidades en los paquetes analizados, área representada exclusivamente en $\mathrm{Klu}$ wer, Science y Taylor.

Destaca, sin duda, la amplitud de la cobertura temática de ScienceDirect que resulta notable incluso en el ámbito de las ciencias puras, que es el que presenta un número inferior de ítems, obviando el área de humanidades, testimonial en este suministrador, ya que su orientación preferente se dirige a las ciencias de la naturaleza y la salud.

Kluwer supone una importante aportación en todas las áreas temáticas, que se vería incrementada si se hubieran contabilizado los e-books. Por lo que se refiere a las publicaciones seriadas, destaca de modo notable en ciencias sociales mientras que la abundante oferta de libros de este distribuidor se adscribe principalmente a las ciencias técnicas, ciencias puras y ciencias de la salud.

\section{«Destaca la amplitud de la cober- tura temática de ScienceDirect que resulta notable incluso en el ámbito de las ciencias puras»}

Taylor \& Francis oferta contenidos de todas las áreas temáticas, resultando reseñable su aportación en el ámbito de las ciencias sociales y, en menor medida, en el área de ciencias de la salud. Por el contrario, resultan escasos los contenidos técnicos suministrados. El campo de las ciencias sociales se vería incrementado de haber contabilizado sus libros electrónicos que cubren mayoritariamente este área además de la de las humanidades.

Resulta equilibrado el reparto de Wiley InterScience que casi alcanza el centenar de publicaciones en el ámbito en que se encuentra en inferioridad de condiciones (las ciencias sociales) destacando en el área de las ciencias técnicas, fundamentalmente por el alto vo- lumen de e-books que oferta de estas disciplinas. Este proveedor carece de contenidos humanísticos.

Opuesto es el caso de Emerald, dirigido con carácter de exclusividad a la distribución de contenidos de ciencias sociales y primordialmente de economía y ciencias de la información. SpringerLink, por el contrario, no tiene incidencia en el campo de las ciencias sociales, destacando en ciencias de la naturaleza, la salud y ciencias puras fundamentalmente. No obstante, este distribuidor dentro de sus series de libros posee un alto volumen de publicaciones unitarias de ciencias técnicas (1.800).

\section{Cobertura vertical (periodo temporal)}

No resulta sencillo realizar con exactitud el recuento conjunto de fechas de inicio de las publicaciones dado que, de un lado, existen contenidos sin fechas $\mathrm{y}$, de otro, se presentan divergencias en las mismas. El panorama se complica debido a la consustancialmente compleja historia de las publicaciones periódicas: revistas cerradas, backfiles, escisiones, fusiones, etc.

Es reseñable el amplio alcance retrospectivo de los contenidos de ScienceDirect, algunas de cuyas publicaciones seriadas se ofertan desde el siglo XIX; tal es el caso de las revistas The lancet de Elsevier, que se inicia en 1823 y del Journal of the Franklin Institute de Pergamon, presente desde 1826. Es significativo el número de títulos de las décadas de los 40, 50 y 60 del siglo pasado: sirva como ejemplo la revista Biochimica et biophysica acta de Elsevier con contenidos desde 1947. A pesar de todo ello, la masiva incorporación de revistas se produce durante los 90, particularmente a partir de 1993. El año 1998 marca el hito en la oferta de este distribuidor.

Destaca la importante integración de contenidos en la década de los 90 también en el resto de suministradores, resultando significativa la incorporación en determinadas fechas como 1994 en el caso de Emerald. La fecha clave de entrada de las revistas de SpringerLink y de Wiley Interscience se sitúa en el año 1996. En el caso de éste último proveedor, a dicho año corresponden como fecha de inicio más de un 55\% del total de las publicaciones abiertas ofrecidas.

El año central en Kluwer Online es 1997 para las publicaciones seriadas. En el importante apartado de libros de esta empresa, en el que se recogen publicaciones desde 1987, el vértice destacado se fija en el año 2002 con un total de 520 títulos.

La oferta de contenidos de Taylor \& Francis se retrotrae a 1995, incrementándose sustancialmente en 1999 para alcanzar su vértice máximo en el año 2000. Sólo existen 9 revistas con contenidos anteriores a 
1995, siendo las más antiguas Journal of modern optics disponible desde 1954 e International journal of radiation biology desde 1959, ambas editadas por Taylor \& Francis. Es reseñable la apuesta de esta empresa en la edición de libros electrónicos cuya presencia se inicia en la década de los 60 , observándose un crecimiento progresivo desde mediados de los años 80, alcanzando el punto álgido en 1999 con 772 títulos incorporados. Se mantiene el ritmo de edición en los primeros años del presente siglo.

\section{«Consideramos mejorable la organización temática de la ma- yoría de los suministradores analizados»}

La importante presencia de contenidos de los primeros años de este milenio igualmente en Wiley InterScience y, en menor medida, en ScienceDirect y SpringerLink obedece a la publicación de colecciones de libros en los que destaca su actualidad.

\section{Discusión}

La evaluación de contenidos presentada permite constatar la extensa cobertura general, la densidad por materias y el alcance retrospectivo de ScienceDirect. El análisis evidencia igualmente una destacada posición de Kluwer y Taylor, reforzada en el apartado de libros electrónicos. La amplitud horizontal de la información es considerable, asimismo, en el paquete de Wiley Interscience. Estos productos, al igual que el de Springer, presentan un carácter enciclopédico, descuidando sólo el ámbito de las humanidades, únicamente presente en Kluwer, Taylor y, en menor medida, en Science. Por el contrario, Emerald destaca por su especialización y orientación a las ciencias sociales.

Consideramos mejorable la organización temática de la mayoría de los suministradores analizados. Sólo SpringerLink presenta unas categorías ajustadas a sus contenidos y bajo unas etiquetas claramente autoexplicativas. Parecería más adecuado un inferior número de clases temáticas en Emerald, Kluwer Online, ScienceDirect y Taylor \& Francis, acudiendo a la subdivisión en subcategorías en caso necesario, solución arbitrada con éxito por Wiley InterScience. Taylor acude a este modelo de jerarquización de contenidos, que resultaría más adecuado con un menor número de categorías principales.

Sería deseable que los suministradores de los paquetes proporcionasen una información más fidedigna acerca de los contenidos cubiertos: publicaciones ofertadas, temática a la que se adscriben, alcance retrospectivo, textos completos o referenciales, formatos disponibles, etc. Resulta difícil para el licenciatario averiguar estos extremos que consideramos prioritarios en la relación contractual establecida.

Juzgamos necesaria la realización de estudios complementarios al presente, que analicen la relevancia científica de los contenidos electrónicos ofertados, el solapamiento existente entre los distribuidores y las facilidades de acceso y funcionalidades de cada uno de los sistemas. Dichos estudios sobre la calidad, así como aquéllos derivados de los datos de uso de los productos de los proveedores de información electrónica, resultarán una aportación empírica imprescindible en el debate internacional sobre los modelos de adquisición de revistas científicas en la biblioteca universitaria del futuro, jugando un papel trascendental en la renegociación de licencias.

\section{Referencias bibliográficas}

Alvite Díez, M. Luisa; Rodríguez Bravo, Blanca."Análisis de la distribución de contenidos electrónicos de Wiley InterScience”. En: El profesional de la información, 2004, mayo-junio, v. 13, n. 3, pp. 209-220.

Alvite Díez, M. Luisa; Rodríguez Bravo, Blanca. “Análisis de la distribución de contenidos electrónicos de ScienceDirect”. En: El profesional de la información, 2004, septiembre-octubre, v. 13, n. 5, pp. 353-361.

Association of Research Libraries. Principles for emerging systems of scholarly publishing, 2000. Consultado en: 02-06-04.

http://www.arl.org/scomm/tempe.html

Bishop, Ann P.; Buttenfield, Barbara P.; Van House, Nancy (eds.). Digital library use: social practice in design and evaluation. Cambridge, MA: The MIT Press, 2003.

Declaración de Valparaíso.

http://www.icsep.info/programa/docs/es/Declaracion_Valparaiso.htm

Eason, Ken; Richardson, Sue; Yu, Liangzhi. "Patterns of use of electronic journals". En: Journal of documentation, 2000, v. 56, n. 5, pp. 477504.

Jewell, Timothy D. Selection and presentation of commercially available electronic resources: issues and practices. Washington, D. C.: Digital Library Federation, 2001. Consultado en: 15-06-04.

http://www.clir.org/pubs/reports/pub99/pub99.pdf

JISC Scholarly Communications Group. Final report from the JISC Scholarly Communications Group (SCG) to the Research Support Libraries Group (RSLG), 2002. Consultado en: 21-05-04.

http://www.jisc.ac.uk/uploaded_documents/rslg.pdf

Pullinger, David. "Academics and the new information environment: the impact of local factors on use of electronic journals". En: Journal of information science, 1999, v. 25, n. 2, pp. 164-172.

Tenopir, Carol. Use and users of electronic library resources: an overview and analysis of recent research studies. Washington, D. C.: Council on Library and Information Resources, 2003. Consultado en: 16-06-04. http://www.clir.org/pubs/reports/pub120/pub120.pdf

Urbano, Cristóbal (dir.). Ús de revistes electròniques de compra consorciada del CBUC (2000-2003). Informe realitzat per encàrrec del Consorci de Biblioteques Universitàries de Catalunya, 2004.

Consultado en: 16-08-04.

http://www.cbuc.es/5digital/Informe_revistes_CBUC_amb_grafics.pdf

Blanca Rodríguez Bravo, Ma Luisa Alvite Díez, Área de Biblioteconomía y Documentación, Facultad de Filosofía y Letras, Universidad de León.

dphbrb@unileon.es

luisa.alvite@unileon.es 